\title{
Depression, anxiety, stress, and their associated factors among Jimma University staff, Jimma, Southwest Ethiopia, 2016: a cross-sectional study
}

This article was published in the following Dove Press journal:

Neuropsychiatric Disease and Treatment

8 November 2017

Number of times this article has been viewed

\author{
Yigizie Yeshaw' \\ Andualem Mossie ${ }^{2}$ \\ 'Department of Medical Physiology, \\ College of Medicine and Health \\ Sciences, University of Gondar, \\ Gondar, ${ }^{2}$ Department of Biomedical \\ Sciences, College of Health Sciences, \\ Jimma University, Jimma, Ethiopia
}

Background: Worldwide, approximately 450 million people suffer from mental disorders. Of these, approximately 150 million are affected with depression. Depression, anxiety, and stress have an impact on productivity, motivation to work, sleep behavior of the individual, and outcome of different chronic diseases. However, till date, there are no studies which evaluated mental health problems among university staff in Ethiopia. Therefore, in this study, we aimed to assess the prevalence of depression, anxiety, stress, and their associated factors among Jimma University staff.

Methods: An institution-based cross-sectional study was conducted among 354 staff of Jimma University from March 24 to April 24, 2016. Stratified simple random sampling technique was used. Pretested interviewer-administered Depression, Anxiety and Stress Scale (DASS-21) questionnaire was used to collect the data. Data were analyzed using SPSS Version 20.0 software.

Results: The prevalence of depression, anxiety, and stress in this study was found to be approximately $22.9 \%, 19.2 \%$, and $28.2 \%$, respectively. Being female (adjusted odds ratio [AOR] $=2.43$, $95 \%$ confidence interval $[\mathrm{CI}]=1.22-4.77)$, no job satisfaction $(\mathrm{AOR}=10.59,95 \% \mathrm{CI}=4.88-22.98)$, presence of conflict with colleagues (AOR $=2.33,95 \% \mathrm{CI}=1.21-4.49$ ), and khat chewing ( $A O R=4.99,95 \%$ CI $=2.57-9.69)$ were associated with depression. Presence of conflict with colleagues $(\mathrm{AOR}=2.46,95 \% \mathrm{CI}=1.25-4.85)$, no job satisfaction $(\mathrm{AOR}=7.12,95 \% \mathrm{CI}=3.29-15.45)$, and khat chewing $(\mathrm{AOR}=2.94,95 \% \mathrm{CI}=1.52-5.66)$ were associated with anxiety. Being widowed $(\mathrm{AOR}=7.46,95 \% \mathrm{CI}=1.11-50.15)$, female $(\mathrm{AOR}=2.72,95 \% \mathrm{CI}=1.40-5.28)$, no job satisfaction $(\mathrm{AOR}=6.69,95 \% \mathrm{CI}=3.46-12.97)$, khat chewing $(\mathrm{AOR}=2.78,95 \% \mathrm{CI}=1.49-5.21)$, and presence of conflict with colleagues ( $\mathrm{AOR}=2.93,95 \% \mathrm{CI}=1.57-5.46$ ) were associated with stress.

Conclusion: The burden of depression, anxiety, and stress among Jimma University staff was found to be high. Being female, widowed, or khat chewer or having a history of conflict with colleagues and no job satisfaction were predictors of depression, anxiety, and stress. Therefore, it is recommended to design preventive strategies to reduce the risk of these problems and to minimize the disease burden.

Keywords: substance use, mental health problems, risk factors, Ethiopia

\section{Background}

Depression, anxiety, and stress are now the major mental health problems that cause disability globally, and no one is immune to these problems. ${ }^{1,2}$ Severe mental health problems interfere with individuals' emotional, cognitive, as well as social abilities that can lead to underemployment and reduced productivity. ${ }^{3,4}$ Depression manifests as loss of interest or pleasure, sadness, feelings of guilt or low self-worth, disturbed sleep or appetite, extreme tiredness, and poor concentration. ${ }^{5}$ It affects
Correspondence: Yigizie Yeshaw Department of Medical Physiology, College of Medicine and Health Sciences, University of Gondar, PO Box 196, Gondar, Ethiopia

Tel +25I 91 8291259

Email yigizieyeshaw29@gmail.com 
performance of job, quality of sleep, routine activities, and productivity of the affected individual. ${ }^{6-9}$ It can also affect the outcome of chronic diseases such as diabetes, cardiovascular diseases, cancer, and obesity. ${ }^{10,11}$ Depression is predicted to be the world's largest health problem by the year 2020. ${ }^{12}$ Worldwide about 450 million people suffer from mental health problems. Of them, about 150 million are affected with depression. ${ }^{1,13}$ According to a Korean study, the prevalence of depression is $26.1 \%$ in men and $28.7 \%$ in women employees. ${ }^{14}$

Anxiety is a response of body to a perceived threat which is triggered by an individual's beliefs, feelings, and thoughts and is characterized by worried thoughts, tension, increased blood pressure, respiratory rate, pulse rate, sweating, difficulty of swallowing, dizziness, and chest pain. ${ }^{15}$ Worldwide about 272.2 million people have anxiety disorder. ${ }^{16}$ It affects about $14.0 \%$ of the European population. ${ }^{17}$

Stress is a feeling that is initiated when a person perceives that demands exceed resources mobilized by the individual. ${ }^{18}$ Prevalence of stress among the staff of the University of Malaysia was found to be $21.7 \%$, which is a major risk factor for various health conditions. ${ }^{19}$ Prevalence of depression, anxiety, and stress among Chinese nurses was found to be $35.8 \%, 37.3 \%$, and $41.1 \%$, respectively. ${ }^{20}$ Another study conducted among 2641 Chinese physicians revealed that $\sim 25.67 \%$ and $28.13 \%$ had anxiety and depression, respectively. ${ }^{21}$ Mental health problems are also common in Africa as indicated by limited studies conducted so far. For example, a study reported the prevalence of mental health problems in a rural district of Kenya as $10.8 \%$, and some other studies demonstrated a prevalence of $27 \%$ in South Africa. ${ }^{22,23}$ A cross-sectional study conducted among medical students in Egypt showed that the prevalence of depression, anxiety, and stress among students was $63.6 \%, 78.4 \%$, and $57.8 \%$, respectively. ${ }^{24}$ However, there is no sufficient research conducted to determine the prevalence of depression, anxiety, and stress in Ethiopia. According to a report of World Health Organization, the prevalence of depression in Ethiopia is $\sim 9.1 \%{ }^{25}$ Furthermore, the prevalence of depression among Jimma town community was found to be $29 \%{ }^{26}$ A research conducted on the prevalence of stress among Jimma University medical students found a prevalence rate of $52.4 \% .{ }^{27}$ Different factors affect the prevalence of depression, anxiety, and stress. Substance use such as chewing khat, drinking alcohol, and cigarette smoking are the major factors that affect these mental health conditions. Chronic nicotine intake affects the brain reward system function which results in depression and anxiety symptoms. ${ }^{28}$ Heavy alcohol consumption is also associated with anxiety disorder. ${ }^{29,30}$ Khat chewers show diversified psychological symptoms including stress, anxiety, depression, and emotional instability; this is due to its content of cathinone and cathine, which causes an increase in the release of catecholamines from presynaptic storage site (sympathomimetic effect). ${ }^{31,32}$ The other determinant factors of depression, anxiety, and stress are work-related factors. Individuals with workplace conflict, prolonged working hours, low job satisfaction, and sleep problems had increased risk of depression, anxiety, and stress. ${ }^{20,21}$ Being a woman, divorced, widowed or separated, and having low socioeconomic status are also sociodemographic determinants of depression, anxiety, and stress. ${ }^{14,33-36}$ On the contrary, a research conducted in the United Kingdom showed that people who had higher salary had high scores of anxiety and depression than those with lower salary. ${ }^{37}$ While these problems have different health effects and exist in the study area, there are no studies performed to evaluate the magnitude of depression, anxiety, and stress among university staff in Ethiopia so far. Therefore, in this study, we aimed to determine the prevalence of these problems and their associated factors among Jimma University staff, so that it can be used as a baseline for subsequent studies in teaching institutions.

\section{Methods}

\section{Study area and population}

This was an institution-based cross-sectional study. The study was performed from March 24 to April 24, 2016, at Jimma University College of Health Sciences, Southwest Ethiopia. All academic and administrative staff of the College of Health Sciences and that of the hospital, who have been working for at least 6 months, were included in the study. Those who were severely ill in the staff or those who were having hearing and speaking difficulty were excluded. Among 3,395 Jimma University College of Health Sciences and Jimma University Hospital staff, 363 were recruited by using simple random sampling after stratification. This means the study population $(3,395)$ was first divided into four strata: academic university staff, administrative university staff, hospital health professionals, and administrative hospital staff. Then, simple random sampling method was employed to select sample population from each stratum.

\section{Ethical consideration}

Ethical clearance was obtained from the Institutional Review Board of Jimma University. A permission letter was obtained from Jimma University Hospital. Written informed consent 
was obtained from each study participant. Confidentiality was maintained.

\section{Data collection}

Data were collected by four psychiatry-trained nurses using pretested interviewer-administered Depression Anxiety Stress Scale 21 (DASS-21) questionnaire. Depression, anxiety, and stress were measured using Lovibond and Lovibond's short version of the DASS- $21 .{ }^{38}$ DASS-2 1 is a psychological screening instrument which is capable of differentiating symptoms of depression, anxiety, and stress. It is a validated and reliable instrument with 21 items in three domains. Each domain comprises seven items assessing symptoms of depression, anxiety, and stress. Participants were asked to indicate the presence of symptoms in each domain over the past week scoring from 0 (did not apply at all) to 3 (applied most of the time). Scores from each dimension were summed. Then, the final score was multiplied by 2 and then categorized according to the DASS manual as normal, mild, moderate, severe, and extremely severe. Accordingly for participants with depression, a depression score of 0-9 was considered normal, 10-13 as mild, 14-20 as moderate, $21-27$ as severe, and 28 and above as extremely severe. For participants with anxiety, an anxiety score of 0-7 was considered normal, 8-9 as mild, 10-14 as moderate, $15-19$ as severe, and 20 and above as extremely severe. For participants with stress, a stress score of $0-14$ was considered normal, 15-18 as mild, 19-25 as moderate, 26-33 as severe, and 34 and above as extremely severe.

\section{Operational definitions}

Khat chewer - the proportion of individuals who had ever used khat at least once in his/her lifetime. Cigarette smokerthe proportion of individuals who had ever used cigarette at least once in his/her lifetime. Alcohol drinker - the proportion of individuals who had ever used alcohol drinks such as tela, tej, katicala/areke, beer, wine, or other drinks that can cause intoxication at least once in his/her lifetime.

\section{Data processing and analysis}

The data collected were checked for completeness and entered into Epi-data version 3.1. Then, the data were exported to Statistical Package for Social Science version 20.0 software for further analysis. Bivariable logistic regression analysis was performed to find the association of each independent variable with the outcome variables. All variables with a $p$-value of $<0.25$ at bivariable logistic regression analysis were entered into the multivariable logistic regression model.
A $p$-value of $<0.05$ was considered statistically significant, and the adjusted odds ratio (AOR) with 95\% confidence interval (CI) was calculated.

\section{Results \\ Sociodemographic characteristics of respondents}

Three hundred and sixty-three subjects were included in the study and the overall response rate was 354 (98.3\%). The majority of respondents were female (201 [56.8\%]). The mean ( \pm standard deviation) age of participants was 29.7 years $( \pm 7.0)$, and the age of the participants was between 20 and 57 years. With regard to religion, $155(43.8 \%)$ respondents were Orthodox Christians and $110(31.1 \%)$ respondents were Muslims. The majority of respondents (194 (54.8\%)) were Oromo followed by Amhara (85 (24\%)). One hundred and sixty-six of them (46.9\%) were married and 158 (44.6\%) were single. One hundred and forty-six respondents $(41.2 \%)$ had a bachelor's degree and 143 (40.4\%) had a diploma. About one-third of the respondents (107 [30.2\%]) earned a monthly income of 3,145 ETB and above followed by $26.6 \%$ with a monthly income in the range of 550-1,114 ETB. Most of them, 151 (42.6\%), were administrative university staff followed by hospital health professionals (77 [21.8\%]) (Table 1).

\section{Work, chronic disease, and substance use-related characteristics of respondents}

More than half of the respondents (186 [52.5\%]) worked in a shift rotation pattern. One hundred and seventeen (33.1\%) respondents reported the presence of conflict with colleagues at workplace in the past 1 month, and 192 (54.2\%) respondents were satisfied with their current job (profession). One hundred and thirty-seven (38.7\%) respondents did not experience an 8-hour sleep per 24 hours in the last month. Eighty-eight (24.9\%) respondents worked more than 8 hours per day in the last month. Only $11(3.1 \%)$ respondents suffered from chronic illness. Of them, $8(2.3 \%)$ respondents had hypertension and the remaining $3(0.8 \%)$ had diabetes mellitus. The overall prevalence of alcohol drinking, khat chewing, and cigarette smoking was found to be 205 (57.9\%), 145 (41\%), and 17 (4.8\%), respectively (Table 2).

\section{Prevalence of depression, anxiety, and stress}

The overall prevalence of depression, anxiety, and stress in this study was found to be $22.9 \%, 19.2 \%$, and $28.2 \%$, respectively. Forty-nine (13.84\%) respondents had mild depression, 
Table I Sociodemographic characteristics of Jimma University staff, Jimma, Ethiopia, 2016

\begin{tabular}{|c|c|}
\hline Variables & Frequency (\%) \\
\hline \multicolumn{2}{|l|}{ Sex } \\
\hline Male & $153(43.2)$ \\
\hline Female & $20 \mathrm{I}(56.8)$ \\
\hline \multicolumn{2}{|l|}{ Religion } \\
\hline Orthodox & $155(43.8)$ \\
\hline Muslim & $110(31.1)$ \\
\hline Protestant & $80(22.6)$ \\
\hline Other ${ }^{\mathrm{a}}$ & $9(2.5)$ \\
\hline \multicolumn{2}{|l|}{ Nationality } \\
\hline Oromo & $194(54.8)$ \\
\hline Amhara & $85(24)$ \\
\hline Gurage & $36(10.2)$ \\
\hline Tigray & $29(8.2)$ \\
\hline Other ${ }^{b}$ & $10(2.8)$ \\
\hline \multicolumn{2}{|l|}{ Age (years) } \\
\hline 18-24 & 7I (20.I) \\
\hline $25-34$ & $223(63.0)$ \\
\hline $35-44$ & $35(9.9)$ \\
\hline$\geq 45$ & $25(7.1)$ \\
\hline \multicolumn{2}{|l|}{ Marital status } \\
\hline Married & $166(46.9)$ \\
\hline Single & $158(44.6)$ \\
\hline Divorced & $19(5.4)$ \\
\hline Widowed & II (3.I) \\
\hline \multicolumn{2}{|l|}{ Educational status } \\
\hline Secondary & $37(10.5)$ \\
\hline Diploma & $143(40.4)$ \\
\hline Degree & $146(41.2)$ \\
\hline$\geq \mathrm{MA} / \mathrm{MSc}$ & $28(7.9)$ \\
\hline \multicolumn{2}{|l|}{ Job } \\
\hline Administrative university staff & $15 \mid(42.6)$ \\
\hline Administrative hospital staff & $65(18.4)$ \\
\hline Hospital health professionals & 77 (2I.8) \\
\hline Academic staff & $61(17.2)$ \\
\hline \multicolumn{2}{|l|}{ Salary (ETB) } \\
\hline $550-1,114$ & $94(26.6)$ \\
\hline $1,115-1,800$ & $88(24.9)$ \\
\hline $\mathrm{I}, 80 \mathrm{I}-3, \mathrm{I} 44$ & $65(18.4)$ \\
\hline$\geq 3,145$ & $107(30.2)$ \\
\hline
\end{tabular}

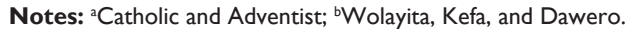
Abbreviation: ETB, Ethiopian Birr.

28 (7.91\%) had moderate depression, and 4 (1.13\%) had severe depression. Similarly, 30 (8.47\%) respondents had mild anxiety, 35 (9.89\%) had moderate anxiety, and $3(0.85 \%)$ had severe anxiety. Forty-nine (13.84\%) respondents had mild stress, 39 (11.02\%) had moderate stress, and $12(3.39 \%)$ had severe stress. The staff members were not severely depressed, anxious, or stressed (Figure 1).

\section{Associated factors of depression}

On bivariable logistic regression analysis, depression was associated with sex, age, marital status, educational status,
Table 2 Work, chronic disease, and substance use-related characteristics of respondents, Jimma, Ethiopia, 2016

\begin{tabular}{ll}
\hline Variables & Frequency (\%) \\
\hline Worked in a shift rotation pattern & \\
Yes & $186(52.5)$ \\
No & $168(47.5)$ \\
Conflict with colleagues & \\
$\quad$ Yes & $117(33.1)$ \\
No & $237(66.9)$ \\
Job satisfaction & \\
Yes & $192(54.2)$ \\
No & $162(45.8)$ \\
Sleeping time/24 hours & \\
$\geq 8$ hours & $217(61.3)$ \\
$<8$ hours & $137(38.7)$ \\
History of chronic disease & \\
Yes & $11(3.1)$ \\
No & $343(96.9)$ \\
Alcohol drinking & \\
Yes & $205(57.9)$ \\
No & $149(42.1)$ \\
Khat chewing & \\
Yes & $145(4 I)$ \\
No & $209(59)$ \\
Cigarette smoking & \\
Yes & $17(4.8)$ \\
No & $337(95.2)$ \\
\hline
\end{tabular}

income, sleep duration, job satisfaction, conflict with colleagues, and substance use (khat chewing, alcohol drinking, and cigarette smoking). In the final model, sex, salary, job satisfaction, conflict with colleagues, and khat chewing were significantly associated with depression $(p<0.05)$. Female staff were 2.4 times more likely to have depression than males (AOR $=2.4,95 \% \mathrm{CI}=1.2-4.9)$. Staff who were not satisfied with their jobs were 10.6 times more likely to have depression than those who were satisfied with their jobs ( $A O R=10.6,95 \% \mathrm{CI}=4.9-23.0)$. Staff who had history of conflict with colleagues in the past 1 month before the study were 2.3 times more likely to develop depression than those who had no history of conflict with their colleagues (AOR $=2.3,95 \% \mathrm{CI}=1.2-4.5$ ). Those staff who earned a salary in the range of 550-1,114 ETB were 0.27 times less likely to have depression than those who earned a salary in the range of $3,145 \mathrm{ETB}$ and above ( $\mathrm{AOR}=0.27$, $95 \% \mathrm{CI}=0.15-0.85)$, and similarly staff who earned a salary in the range of 1,801-3,144 ETB were 0.35 times less likely to have depression than those who earned a salary in the range of 3,145 ETB and above (AOR $=0.35,95 \%$ $\mathrm{CI}=0.11-0.68)$. Khat chewer staff were 5.0 times more likely to have depression than nonchewers $(\mathrm{AOR}=5.0,95 \%$ $\mathrm{CI}=2.6-9.7$ ) (Table 3). 


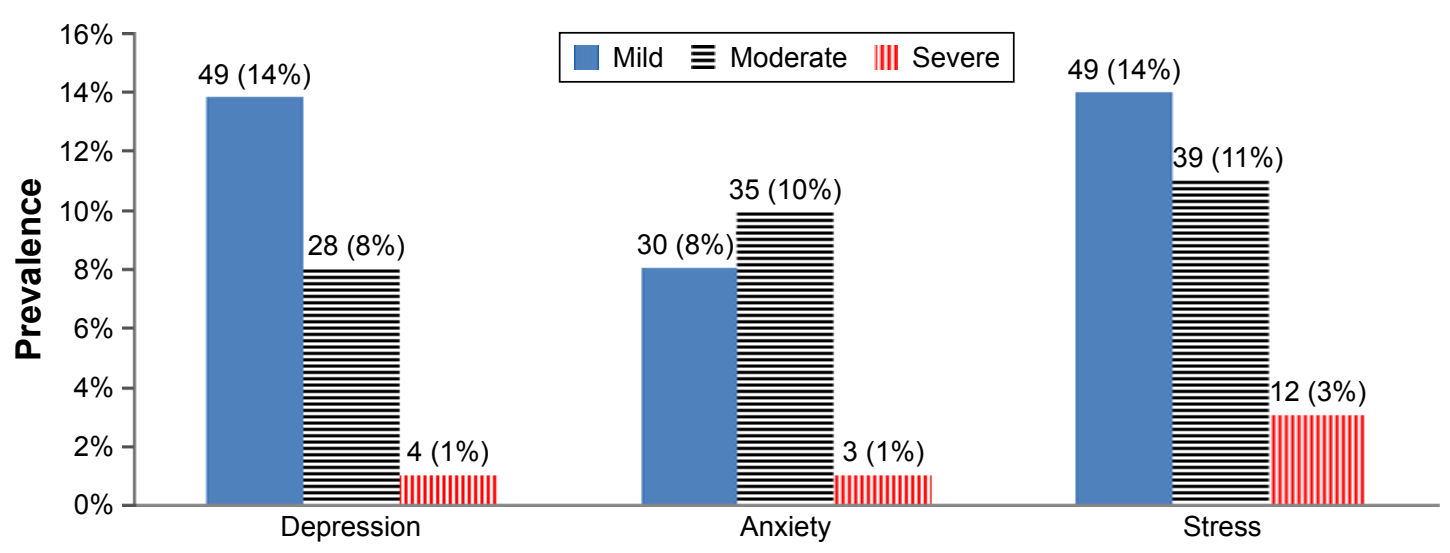

Level of depression, anxiety, and stress

Figure I Severity of depression, anxiety, and stress among Jimma University staff, Jimma, Ethiopia, 2016.

Table 3 Factors associated with depression among Jimma University staff: logistic regression analysis, Jimma, Ethiopia, 2016

\begin{tabular}{|c|c|c|c|c|}
\hline \multirow[t]{3}{*}{ Variables } & \multicolumn{2}{|c|}{ Depression } & \multicolumn{2}{|l|}{ OR } \\
\hline & Yes & No & COR $(95 \% \mathrm{Cl})$ & AOR (95\% Cl) \\
\hline & $\mathbf{N}(\%)$ & $\mathbf{N}(\%)$ & & \\
\hline \multicolumn{5}{|l|}{ Sex } \\
\hline Male & $29(19.0)$ & I $24(8 \mid .0)$ & 1.00 & \\
\hline Female & $52(25.9)$ & $149(74.1)$ & $1.49(0.89-2.49)$ & $2.43(1.22-4.87)^{*}$ \\
\hline \multicolumn{5}{|l|}{ Age (years) } \\
\hline 18-24 & 14 (19.7) & $57(80.3)$ & 1.00 & \\
\hline $25-34$ & $46(20.6)$ & $177(79.4)$ & $1.06(0.54-2.07)$ & $0.63(0.27-1.45)$ \\
\hline $35-44$ & $15(42.9)$ & $20(57.1)$ & $3.05(1.26-7.43)$ & $1.21(0.38-3.90)$ \\
\hline$\geq 45$ & $6(24.0)$ & $19(76.0)$ & $1.29(0.43-3.82)$ & $0.4 \mid(0.1 \mathrm{I}-\mathrm{I} .58)$ \\
\hline \multicolumn{5}{|l|}{ Marital status } \\
\hline Married & $24(14.5)$ & $142(85.5)$ & 1.00 & \\
\hline Single & $4 I(25.9)$ & 117 (74.9) & $2.07(1.18-3.63)$ & I. $10(0.53-2.28)$ \\
\hline Divorced & $9(47.4)$ & $10(52.6)$ & $5.33(1.96-14.46)$ & $2.98(0.69-12.81)$ \\
\hline Widowed & 7 (63.6) & $4(36.4)$ & $10.35(2.82-38.08)$ & $3.18(0.59-17.03)$ \\
\hline \multicolumn{5}{|c|}{ Educational status } \\
\hline Secondary & $5(13.5)$ & $32(86.5)$ & $0.24(0.07-0.8 I)$ & $1.72(0.26-11.26)$ \\
\hline Diploma & $31(2 \mid .7)$ & $112(78.3)$ & $0.43(0.18-1.01)$ & I.87 (0.42-8.29) \\
\hline Degree & $34(23.3)$ & 112 (76.7) & $0.47(0.20-1.10)$ & $2.42(0.74-7.9 I)$ \\
\hline$\geq \mathrm{MA} / \mathrm{MSc}$ & II (39.3) & $17(60.7)$ & 1.00 & \\
\hline \multicolumn{5}{|l|}{ Salary (ETB) } \\
\hline 550-I,II4 & $15(16.0)$ & $79(84.0)$ & $0.39(0.20-0.77)$ & $0.27(0.15-0.85)^{*}$ \\
\hline $1,115-1,800$ & $20(22.7)$ & $68(77.3)$ & $0.6 \mid(0.32-1.15)$ & $0.68(0.30-1.55)$ \\
\hline $\mathrm{I}, 80 \mathrm{I}-3, \mathrm{I} 44$ & II (16.9) & $54(83.1)$ & $0.42(0.20-0.90)$ & $0.35(0.1 \mathrm{I}-0.68)^{*}$ \\
\hline$\geq 3,145$ & $35(32.7)$ & $72(67.3)$ & 1.00 & \\
\hline \multicolumn{5}{|l|}{ Job satisfaction } \\
\hline Yes & $10(5.2)$ & I $82(94.8)$ & 1.00 & 1.00 \\
\hline No & 7I (43.8) & 91 (56.2) & 14.20 (6.99-28.83) & $10.59(4.88-22.98)^{* *}$ \\
\hline \multicolumn{5}{|c|}{ Conflict with colleagues } \\
\hline Yes & $50(42.7)$ & $67(57.3)$ & $4.96(2.93-8.39)$ & $2.33(1.21-4.49)^{*}$ \\
\hline No & $31(13.1)$ & $206(86.9)$ & 1.00 & 1.00 \\
\hline \multicolumn{5}{|c|}{ Alcohol drinking } \\
\hline Yes & $59(28.8)$ & I46 (7I.2) & $2.33(1.35-4.02)$ & I.6I (0.79-3.29) \\
\hline No & $22(14.8)$ & $127(85.2)$ & 1.00 & 1.00 \\
\hline \multicolumn{5}{|c|}{ Cigarette smoking } \\
\hline Yes & $6(35.3)$ & II (64.7) & $1.91(0.68-5.32)$ & $0.45(0.13-1.50)$ \\
\hline No & $75(22.3)$ & $262(77.7)$ & 1.00 & 1.00 \\
\hline \multicolumn{5}{|l|}{ Khat chewing } \\
\hline Yes & $54(37.2)$ & $91(62.8)$ & $4.00(2.36-6.77)$ & $4.99(2.57-9.69)^{* *}$ \\
\hline No & 27 (12.9) & I $82(87.1)$ & 1.00 & 1.00 \\
\hline
\end{tabular}

Notes: *Statistically significant $(p<0.05)$; **statistically highly significant $(p<0.0 \mathrm{I})$.

Abbreviations: $\mathrm{AOR}$, adjusted odds ratio; $\mathrm{Cl}$, confidence interval; $\mathrm{COR}$, crude odds ratio; MA, master of arts; MSc, master of science. 


\section{Associated factors of anxiety}

On bivariable analysis, anxiety was found to be associated with age, educational status, marital status, salary, duration of sleeping time, job satisfaction, conflict with colleagues, alcohol use, and khat chewing. However, salary, khat chewing, conflict with colleagues, and job satisfaction of respondents were significantly associated with anxiety at the final model. Those staff who were not satisfied with their job were 7.1 times more likely to have anxiety than those who were satisfied with their job (AOR $=7.1,95 \%$ $\mathrm{CI}=3.3-15.5$ ). Staff who had a history of conflict with their colleagues in the past 1 month before the study were 2.5 times more likely to develop anxiety than those who had no history of conflict (AOR $=2.5,95 \% \mathrm{CI}=1.3-4.8$ ). Khat chewers were 2.9 times more likely to develop anxiety than nonchewers $(\mathrm{AOR}=2.94,95 \% \mathrm{CI}=1.52-5.66)$. Participants who earned a salary in the range of 1,801-3,144 ETB were 0.19 times less likely to have anxiety than those who earned a salary of 3,145 ETB and above (AOR $=0.19,95 \%$ $\mathrm{CI}=0.07-0.54)$. Staff who earned a salary in the range of 550-1,114 ETB were 0.41 times less likely to have anxiety than those who earned a salary in the range of 3,145 and above (AOR $=0.41,95 \% \mathrm{CI}=0.17-0.99)$ (Table 4).

\section{Associated factors of stress}

On bivariable logistic regression analysis, stress was associated with age, sex, marital status, educational status, salary, duration of sleep, job satisfaction, conflict with colleagues, alcohol use, khat chewing, and cigarette smoking. On multiple regression analysis sex, marital status, salary,

Table 4 Factors associated with anxiety among Jimma University staff: logistic regression analysis, Jimma, Ethiopia, 2016

\begin{tabular}{|c|c|c|c|c|}
\hline \multirow[t]{3}{*}{ Variables } & \multicolumn{2}{|l|}{ Anxiety } & \multicolumn{2}{|l|}{ OR } \\
\hline & Yes & No & COR (95\% Cl) & AOR (95\% Cl) \\
\hline & $\mathbf{N}(\%)$ & $\mathbf{N}(\%)$ & & \\
\hline \multicolumn{5}{|l|}{ Age (years) } \\
\hline $18-24$ & $12(16.9)$ & $59(83.1)$ & 1.00 & 1.00 \\
\hline $25-34$ & $34(15.2)$ & $189(84.4)$ & $0.88(0.43-1.19)$ & $0.57(0.25-1.32)$ \\
\hline $35-44$ & $16(45.7)$ & $19(54.3)$ & $4.14(1.67-10.28)$ & $2.34(0.78-7.04)$ \\
\hline$\geq 45$ & $6(24.0)$ & $19(76.0)$ & $1.55(0.5 \mid-4.70)$ & $0.73(0.20-2.62)$ \\
\hline \multicolumn{5}{|l|}{ Marital status } \\
\hline Married & $29(17.5)$ & $137(82.5)$ & 1.00 & 1.00 \\
\hline Single & $28(17.7)$ & $130(82.3)$ & $1.02(0.57-1.80)$ & $0.45(0.21-0.95)$ \\
\hline Divorced & $7(36.8)$ & $12(63.2)$ & $2.76(0.10-7.60)$ & $0.82(0.20-3.35)$ \\
\hline Widowed & $4(36.4)$ & $7(63.6)$ & $2.70(0.74-9.83)$ & $0.48(0.10-2.33)$ \\
\hline \multicolumn{5}{|l|}{ Education status } \\
\hline Secondary & $3(8.1)$ & $34(91.90)$ & $0.09(0.22-0.36)$ & $0.29(0.04-1.98)$ \\
\hline Diploma & $27(18.9)$ & $116(8 I .1)$ & $0.23(0.10-0.55)$ & $0.73(0.17-3.19)$ \\
\hline Degree & $24(16.4)$ & $122(83.6)$ & $0.20(0.08-0.47)$ & $0.48(0.15-1.48)$ \\
\hline$\geq \mathrm{MA} / \mathrm{MSc}$ & $14(50.0)$ & $14(50.0)$ & 1.00 & 1.00 \\
\hline \multicolumn{5}{|l|}{ Salary (ETB) } \\
\hline $550-I, I 14$ & $12(12.8)$ & $82(87.2)$ & $0.36(0.17-0.75)$ & $0.4 \mathrm{I}(0.17-0.99)^{*}$ \\
\hline$I,|1| 5-1,800$ & $18(20.5)$ & $70(79.5)$ & $0.63(0.32-1.23)$ & $0.84(0.38-1.87)$ \\
\hline $\mathrm{I}, 80 \mathrm{I}-3, \mathrm{I} 44$ & $7(10.8)$ & $58(89.2)$ & $0.30(0.12-0.72)$ & $0.19(0.07-0.54)^{*}$ \\
\hline$\geq 3,145$ & $31(29.0)$ & $76(71.0)$ & 1.00 & 1.00 \\
\hline \multicolumn{5}{|c|}{ Sleeping time $/ 24 \mathrm{hrs}$} \\
\hline$\geq 8$ hours & 34 (I5.7) & $183(84.3)$ & 1.00 & 1.00 \\
\hline$<8$ hours & $34(24.8)$ & $103(75.2)$ & $1.78(1.04-3.03)$ & $1.28(0.65-2.52)$ \\
\hline \multicolumn{5}{|l|}{ Job satisfaction } \\
\hline Yes & $10(5.2)$ & $182(94.8)$ & 1.00 & 1.00 \\
\hline No & $58(35.8)$ & $104(64.2)$ & $10.15(4.98-20.71)$ & $7.12(3.29-15.45) * *$ \\
\hline \multicolumn{5}{|c|}{ Conflict with colleagues } \\
\hline Yes & $42(35.9)$ & $75(64.1)$ & $4.55(2.61-7.92)$ & $2.46(1.25-4.85)^{*}$ \\
\hline No & $26(11.0)$ & $211(89.0)$ & 1.00 & 1.00 \\
\hline \multicolumn{5}{|l|}{ Alcohol drinking } \\
\hline Yes & $44(21.5)$ & I6I (78.5) & $\mathrm{I} .42(0.82-2.47)$ & $0.69(0.3 \mathrm{I}-1.53)$ \\
\hline No & $24(16.1)$ & $125(83.9)$ & 1.00 & 1.00 \\
\hline \multicolumn{5}{|l|}{ Khat chewing } \\
\hline Yes & $43(29.7)$ & $102(70.3)$ & $3.10(1.79-5.37)$ & $2.94(1.52-5.66)^{*}$ \\
\hline No & $25(12.0)$ & $184(88.0)$ & 1.00 & 1.00 \\
\hline
\end{tabular}

Notes: *Statistically significant $(p<0.05)$; **statistically highly significant $(p<0.01)$.

Abbreviations: $\mathrm{AOR}$, adjusted odds ratio; $\mathrm{Cl}$, confidence interval; $\mathrm{COR}$, crude odds ratio; $\mathrm{MA}$, master of arts; $\mathrm{MSc}$, master of science. 
conflict with colleagues, job satisfaction, and khat chewing were significantly associated with stress. Female respondents were 2.7 times more likely to develop stress than males ( $\mathrm{AOR}=2.7,95 \% \mathrm{CI}=1.4-5.3)$. Widowed respondents were 7.5 times more likely to have stress than married once (AOR $=7.5,95 \% \mathrm{CI}=1.1-50.2$ ). Participants who were not satisfied with their work were 6.7 times more likely to have stress than those satisfied with their work (AOR $=6.7,95 \%$ $\mathrm{CI}=3.5-13.0$. Khat chewer staff were 2.8 times more likely to have stress than nonchewers $(\mathrm{AOR}=2.8,95 \% \mathrm{CI}=1.5-5.2)$.
Participants who had conflict with their colleagues in the workplace were 2.9 times more likely to have stress (AOR $=2.9,95 \% \mathrm{CI}=1.6-5.5)$ than those who did not. Respondents who earned a salary in the range of 550-1,114 ETB were 0.44 times less likely to have stress than those who earned a salary of 3,145 ETB and above (AOR $=0.44$, $95 \% \mathrm{CI}=0.19-0.99)$. Similarly, respondents with a salary in the range of 1,801-3,144 ETB were 0.34 times less likely to have stress than those who earned a salary of 3,145 ETB and above $(\mathrm{AOR}=0.34,95 \% \mathrm{CI}=0.14-0.82)$ (Table 5).

Table 5 Factors associated with stress among Jimma University staff: logistic regression analysis, Jimma, Ethiopia, 2016

\begin{tabular}{|c|c|c|c|c|}
\hline \multirow[t]{3}{*}{ Variables } & \multicolumn{2}{|l|}{ Stress } & \multicolumn{2}{|l|}{ OR } \\
\hline & Yes & No & COR (95\% Cl) & AOR (95\% CI) \\
\hline & $\mathbf{N}(\%)$ & $\mathbf{N}(\%)$ & & \\
\hline \multicolumn{5}{|l|}{ Sex } \\
\hline Male & $32(20.9)$ & 121 (79.I) & 1.00 & 1.00 \\
\hline Female & $68(33.8)$ & $133(66.2)$ & $1.93(1.19-3.15)$ & $2.72(1.40-5.28)^{*}$ \\
\hline \multicolumn{5}{|l|}{ Age (years) } \\
\hline $18-24$ & $18(25.4)$ & $53(74.6)$ & 1.00 & 1.00 \\
\hline $25-34$ & $55(24.7)$ & $168(75.3)$ & $0.96(0.52-1.78)$ & $0.70(0.3|-| .53)$ \\
\hline $35-44$ & $18(51.4)$ & $17(48.6)$ & $3.12(1.33-7.31)$ & I.0I (0.30-3.43) \\
\hline$\geq 45$ & $9(36.0)$ & $16(64.0)$ & $1.66(0.62-4.40)$ & 051 (0.13-199) \\
\hline \multicolumn{5}{|l|}{ Marital status } \\
\hline Married & $30(18.1)$ & $136(81.9)$ & 1.00 & 1.00 \\
\hline Single & $50(31.6)$ & 108 (68.4) & $2.10(1.25-3.52)$ & $1.46(0.78-2.75)$ \\
\hline Divorced & II (57.9) & $8(42.1)$ & $6.23(2.31-16.82)$ & $3.61(0.96-13.55)$ \\
\hline Widowed & $9(8 \mathrm{I} .8)$ & $2(18.2)$ & 20.40 (4.19-99.28) & $7.46(1.11-50.15)^{*}$ \\
\hline \multicolumn{5}{|c|}{ Educational status } \\
\hline Secondary & $7(18.9)$ & $30(8 I .1)$ & $1.66(0.68-4.09)$ & $1.30(0.22-7.68)$ \\
\hline Diploma & $40(28.0)$ & $103(72.0)$ & $1.62(0.66-3.98)$ & $\mathrm{I} .50(0.36-6.24)$ \\
\hline Degree & $40(27.4)$ & $106(72.6)$ & 3.71 (I.23-II.25) & $1.63(0.52-5.12)$ \\
\hline$\geq \mathrm{MA} / \mathrm{MSc}$ & I $3(46.4)$ & $15(53.6)$ & 1.00 & 1.00 \\
\hline \multicolumn{5}{|l|}{ Salary (ETB) } \\
\hline 550-I,II4 & $20(21.3)$ & $74(78.7)$ & $0.45(0.24-0.85)$ & $0.44(0.19-0.10)^{*}$ \\
\hline I,II5-I,800 & $25(28.4)$ & $63(71.6)$ & $0.67(0.36-1.22)$ & $0.74(0.33-1.69)$ \\
\hline $\mathrm{I}, 80 \mathrm{I}-3, \mid 44$ & $15(23.1)$ & $50(76.9)$ & $0.50(0.25-1.01)$ & $0.34(0.14-0.82)^{*}$ \\
\hline$\geq 3,145$ & $40(37.4)$ & $67(62.6)$ & 1.00 & 1.00 \\
\hline \multicolumn{5}{|c|}{ Sleeping time/24 hrs } \\
\hline$\geq 8$ hours & $5 \mathrm{I}(23.5)$ & $166(76.5)$ & 1.00 & 1.00 \\
\hline$<8$ hours & $49(35.8)$ & $88(64.2)$ & $0.55(0.35-0.88)$ & $1.28(0.68-2.40)$ \\
\hline \multicolumn{5}{|l|}{ Job satisfaction } \\
\hline Yes & $17(8.9)$ & $175(91.1)$ & 1.00 & 1.00 \\
\hline No & $83(51.2)$ & 79 (48.8) & $10.82(6.02-19.42)$ & $6.69(3.46-12.97)^{* *}$ \\
\hline \multicolumn{5}{|c|}{ Conflict with colleagues } \\
\hline Yes & $62(53.0)$ & $55(47.0)$ & $5.90(3.57-9.75)$ & $2.93(1.57-5.46)^{*}$ \\
\hline No & $38(16.0)$ & $199(84.0)$ & 1.00 & 1.00 \\
\hline \multicolumn{5}{|l|}{ Alcohol drinking } \\
\hline Yes & $69(33.7)$ & $136(66.3)$ & $1.93(1.18-3.15)$ & $1.35(0.69-2.66)$ \\
\hline No & $31(20.8)$ & II 8 (79.2) & 1.00 & 1.00 \\
\hline \multicolumn{5}{|c|}{ Cigarette smoking } \\
\hline Yes & $8(47.1)$ & $9(52.9)$ & $2.37(0.89-6.32)$ & $0.49(0.13-1.8 I)$ \\
\hline No & $92(27.3)$ & $245(72.7)$ & 1.00 & 1.00 \\
\hline \multicolumn{5}{|l|}{ Khat chewing } \\
\hline Yes & $59(40.7)$ & $86(59.3)$ & $2.81(1.75-4.52)$ & $2.78(I .49-5.21)^{*}$ \\
\hline No & $4 \mid(19.6)$ & $168(80.4)$ & 1.00 & 1.00 \\
\hline
\end{tabular}

Notes: *Statistically significant $(p<0.05)$; **statistically highly significant $(p<0.0 \mathrm{I})$.

Abbreviations: $\mathrm{AOR}$, adjusted odds ratio; $\mathrm{Cl}$, confidence interval; $\mathrm{COR}$, crude odds ratio; MA, master of arts; MSc, master of science. 


\section{Discussion}

The overall prevalence of depression in this study was found to be $22.9 \%$, which is almost similar to study conducted on Chinese physicians (28.13\%). ${ }^{21}$ However, the prevalence of depression in this study was less than that reported in a study conducted on hospital nurses in China (35.8\%). ${ }^{20}$ The possible reason for this discrepancy might be the difference in study population. The prevalence of depression in this study was also less than that among medical students of Egypt using the same instrument (questionnaire) (63.6\%). ${ }^{24}$ This difference might be due to stressful academic-related conditions among medical students. The prevalence of depression among Jimma town community has been reported to be about $29 \%$, which is greater than the findings in our study. ${ }^{26}$ This discrepancy might be due to the type of diagnostic instrument used, study population, and socioeconomic differences between university staff and general community. In this study, the prevalence of depression in females (52 [25.9\%]) was greater than that in males $(29(19.0 \%))$. This finding was almost found to be similar by a study conducted on Korean employees (26.1\% for males and $28.7 \%$ for females). ${ }^{14}$

The prevalence of anxiety in this study was found to be $19.2 \%$, which was less than the study conducted on Chinese physicians $(25.67 \%),{ }^{21}$ nurses in China $(37.3 \%),{ }^{20}$ and first-year medical students of Egypt (78.4\%). ${ }^{24}$ This difference might be because nurses and physicians might have increased workload in giving different health services to their patients, which might lead to increased level of anxiety compared to other professions. Similarly, medical students might have excessive working hours, competitive academic environment, staying away from home, and financial problems. This justification was supported by a study conducted among medical students of Egypt which identifies perceived low socioeconomic status, living away from the family, and lack of support from families as some of the risk factors for depression in medical students. ${ }^{24}$ In this study, staff with no job satisfaction and experiencing conflict with colleagues was significantly associated with depression and anxiety. This finding agrees with the studies conducted among Chinese nurses and physicians in that those health professionals who experience workplace conflict and low job satisfaction had increased level of anxiety and depression compared to those who did not encounter. ${ }^{20,21}$ The possible reason is that stressful conditions (low job satisfaction and conflict with colleagues) increase the risk of having stress episodes, which might increase the secretion of adrenocortical hormone, that is, cortisol. If the stressful conditions are not controlled, excess secretion of cortisol for prolonged period damages neurons of the hippocampus and amygdala, which are the important structures of the brain that regulate mood and pleasure which results in depression and anxiety. Females were 2.43 times more likely to have depression than males, and this finding was in line with many different studies. ${ }^{33-36}$ This might be because females are more prone to stressful conditions either due to hormonal changes that occur during pregnancy, postpartum, and postmenopausal periods or due to lack of support from their partner or family. Those all conditions might increase the prevalence of depression among women than among men.

The prevalence of khat chewing in this study was found to be $145(41 \%)$. Chewer staff were 4.98 times more likely to have depression and 2.94 times more likely to have anxiety than nonchewer staff. This finding is in line with other study. ${ }^{31}$ The possible reason is that khat stimulates adrenocortical function. Cathinone and cathine, the primary psychoactive ingredients of khat, stimulate the release of the cortisol, norepinephrine, and dopamine. Consequently, the respondents experience psychostimulatory effects such as excitement and talkativeness initially. Then, they develop excessive worry, depressed mood, and tension. ${ }^{32}$ The other possible justification is the socioeconomic problems caused due to increased demand of money to buy khat.

In this study, those staff who earned a salary of less than 3,145 ETB had reduced risk for depression and anxiety than those who earned a salary of 3,145 ETB and above. This finding is in contrast to that of many other studies. ${ }^{33-36}$ This difference might be due to the placement of those respondents with master's degree in managerial posts (who had higher salary than lower education workers), their depression and anxiety was higher level. This justification is supported by a research conducted in the United Kingdom in which staff with increased salary had high scores of depression and anxiety than those who earned a lower salary. ${ }^{37}$

The prevalence of stress in this study was found to be $28.2 \%$, which agrees with a previous study conducted on Malaysian University staff $(21.7 \%) .{ }^{19}$ However, this result was much lower than the prevalence of stress found in a study conducted on medical students of Jimma University (52.4\%), ${ }^{27}$ in a study conducted on medical students of Egypt (57.8\%), ${ }^{24}$ and in a study conducted on nurses in China (41.1\%). ${ }^{20}$ This difference might be due to similar reasons mentioned in case of depression and anxiety. In this study, respondents who had khat chewing habit, conflict with colleagues at workplace, no job satisfaction, widowed, and being female were significantly associated with stress. This finding is in line with previous studies based on Malaysian and Chinese 
staff. ${ }^{19,20}$ Widowed staff were found to be 7.46 times more likely to have stress than at least once-married staff. This finding is in line with the study conducted on Malaysian staff. ${ }^{19}$ The increased prevalence of stress among widowed staff might be due to the loss of their partners resulting in more stressful conditions.

\section{Conclusion}

This study demonstrated that depression, anxiety, and stress were moderate health problems of Jimma University hospital and university staff. All of those disorders were found to be more common among khat chewers, those with no job satisfaction, and those who had conflict with their colleagues at the workplace. Depression and stress were more common in females. Prevalence of stress was also more common in widowed staff. Therefore, it is recommended to design preventive strategies to reduce the risk of depression, anxiety, and stress and minimize the disease burden.

\section{Limitation of the study}

Study design may not allow to establish cause-and-effect relationships among the variables. Thus, it is not possible to identify whether depression, anxiety, and stress influenced the associated factors or vice versa. In addition, DASS 21 instrument is used only for screening purpose, so it is impossible to reach at diagnosis using this instrument.

\section{Availability of data and materials}

All data and materials related to the study can be obtained through contacting the corresponding author.

\section{Acknowledgments}

We acknowledge Jimma University for its general support to conduct this study. We also acknowledge our data collectors for their role in collecting all necessary information. There was no specific funding for the research.

\section{Author contributions}

All authors contributed toward data analysis, drafting and critically revising the paper and agree to be accountable for all aspects of the work.

\section{Disclosure}

The authors report no conflicts of interest in this work.

\section{References}

1. World Health Organization. Department of Mental Health and Substance Dependence; 2003. Available from: http://www.who.int/mental_health/ media/investing_mnh.pdf
2. World Health Organization. Substance Abuse Dept. Global status report: alcohol policy. World Health Organization; 2004. Available from: http:// www.who.int/substance_abuse/publications/en/Alcohol\%20Policy\%20 Report.pdf

3. McLachlan R, Gilfillan G, Gordon J. Deep and persistent disadvantage in Australia. Australian Government Productivity Commission Staff Working Paper. Australian Government Productivity Commission, Canberra, Australia; 2013;1-236.

4. Slade T, Johnston A, Teesson M, et al. The mental health of Australians 2. Report on the 2007 National Survey of Mental Health and Wellbeing. 2007;20(07). Available from: http://www.health.gov.au/internet/main/ publishing.nsf/\%20content/A24556C814804A99CA257BF0001CAC4 5/\$File/mhaust2.pdf

5. Marcus M, Yasamy MT, van Ommeren M, Chisholm D, Saxena S Depression: A global public health concern. WHO, Department of Mental Health and Substance Abuse. 2012; 1:6-8. Available from: http://www.who.int/mental_health/management/depression/who_ paper_depression_wfmh_2012.pdf

6. Adler DA, Mclaughlin TJ, Rogers WH, Chang H, Lapitsky L, Lerner D Job performance deficits due to depression. Am J Psychiatry. 2006; 163(9):1569-1576.

7. Gilmour H, Patten SB. Depression and work impairment. Health Rep. 2007;18(1):9-22.

8. Ivanova JI, Birnbaum HG, Kidolezi Y, Subramanian G, Khan SA, Stensland MD. Direct and indirect costs of employees with treatmentresistant and non-treatment-resistant major depressive disorder. Curr Med Res Opin. 2010;26(10):2475-2484.

9. Patel V. Cultural factors and international epidemiology. Br Med Bull. 2001;57:33-45.

10. Demyttenaere K, Bruffaerts R, Posada-Villa J, et al; WHO World Mental Health Survey Consortium. Prevalence, severity, and unmet need for treatment of mental disorders in the World Health Organization World Mental Health Surveys. JAMA. 2004;291(21):2581-2590.

11. Mirza I, Jenkins R. Risk factors, prevalence, and treatment of anxiety and depressive disorders in Pakistan: systematic review. BMJ. 2004; 328(7443):794.

12. Murray CJ, Lopez AD. The global burden of disease: a comprehensive assessment of mortality and disability from deceases, injuries and risk factors in 1990 and projected to 2010. Harvard School of Public Health, Harvard University Press. 1996;1:1-35.

13. Lazarus Ray, Freeman M. Primery-level mental health care for commenmental disorders in resource-poor settings: a litrature review. Medical Research Council. 2009.

14. Park SG, Min KB, Chang SJ, Kim HC, Min JY. Job stress and depressive symptoms among Korean employees: the effects of culture on work. Int Arch Occup Environ Health. 2009;82(3):397-405.

15. American Psychiatric Association. Diagnostic and statistical manual of mental disorders, Fifth Edition. (DSM-5®). American Psychiatric Pub; 2013.

16. Baxter AJ, Vos T, Scott KM, Norman RE. The regional distribution of anxiety disorders: implications for the Global Burden of Disease Study. Int J Methods Psychiatr Res. 2014;23(4):422-438.

17. Wittchen HU, Jacobi F, Rehm J, et al. The size and burden of mental disorders and other disorders of the brain in Europe 2010. Eur Neuropsychopharmacol. 2011;21(9):655-679.

18. Usman A, Ahmed Z, Ahmed I, Akbar Z. Work stress experienced by the teaching staff of University of the Punjab, Pakistan: antecedents and consequences. Int J Bus Soc Sci. 2011;2(8):202-210.

19. Mukosolu O, Ibrahim F, Rampal L, Ibrahim N. Prevalence of job stress and its associated factors among Universiti Putra Malaysia staff. Malays J Med Health Sci. 2015;11(1):27-38.

20. Cheung T, Yipaul S. Depression, anxiety and symptoms of stress among Hong Kong nurses: a cross-sectional study. Int J Environ Res Publ Health. 2015;12(9):11072-11100

21. Gong Y, Han T, Chen W, et al. Prevalence of anxiety and depressive symptoms and related risk factors among physicians in China: a crosssectional study. PLoS One. 2014;9(7):1-8. 
22. Havenaar MJ, Geerlings IM, Vivian L, Collinson M, Robertson B. Common mental health problems in historically disadvantaged urban and rural communities in South Africa: prevalence and risk factors. Soc Psychiatry Psychiatr Epidemiol. 2008;43(3):209-215.

23. Jenkins R, Njenga F, Okonji M, et al. Prevalence of common mental disorders in a rural district of Kenya, and socio-demographic risk factors. Int J Environ Res Public Health. 2012;9(5):1810-1819.

24. Abdallah AR, Gabr HM. Depression, anxiety and stress among first year medical students in an Egyptian public university. Int Res J Med Sci. 2014;2(1):11-19.

25. Hailemariam S, Tessema F, Asefa M, Tadesse H, Tenkolu G. The prevalence of depression and associated factors in Ethiopia: findings from the National Health Survey. Int J Ment Health Syst. 2012;6(1):23.

26. Andualem M, Dagmawi K, Alemayehu N. Prevalence and severity of depression and its association with substance use in Jimma town, Southwest Ethiopia. Hindawi J Depress Res Treat. 2016;2016: $1-7$.

27. Leta M, Andualem M, Alemayehu N. Stress among medical students and its association with substance use and academic performance. J Biomed Educ. 2015;2015:1-9.

28. Souza MSD, Markou A. Role of GABA and glutamate circuitry. Cold Spring Harb Perspect Med. 2013;3:1-18.

29. Martín-Merino E, Ruigómez A, Wallander MA, Johansson S, GarcíaRodríguez LA. Prevalence, incidence, morbidity and treatment patterns in a cohort of patients diagnosed with anxiety in UK primary care. Fam Pract. 2010;27(1):9-16.
30. Strine TW, Mokdad AH, Balluz LS, et al. Depression and anxiety in the United States: findings from the 2006 Behavioral Risk Factor Surveillance System. Psychiatr Serv. 2008;59(12):1383-1390.

31. Odenwald $\mathrm{M}$, Khatkonsum C. Chronic khat use and psychotic disorders: a review of the literature and future prospects. SUCHT. 2008;53(1): 9-22.

32. Cox G. Adverse effects of khat: a review. Adv Psychiatr Treat. 2003; 9(6):456-463.

33. Kaneita Y, Ohida T, Uchiyama M, et al. The relationship between depression and sleep disturbances: a Japanese nationwide general population survey. J Clin Psychiatry. 2006;67(2):196-203.

34. Welsh D. Predictors of depressive symptoms in female medical-surgical hospital nurses. Issues Mental Health Nurs. 2009;30(5):320-326.

35. Ivbijaro G, Garrison P, Funk M. Mental health in primary care gap: now is the time to act. Ment Health Fam Med. 2009;6(1):1-4.

36. Weyerer S, Eifflaender-Gorfer S, Köhler L, et al; German AgeCoDe Study group (German Study on Ageing, Cognition and Dementia in Primary Care Patients). Prevalence and risk factors for depression in non-demented primary care attenders aged 75 years and older. $J$ Affect Disord. 2008;111(2-3):153-163.

37. Caplan RP. Stress, anxiety, and depression in hospital consultants, general practitioners, and senior health service managers. BMJ. 1994; 309(6964):1261-1263.

38. Lovibond SH, Lovibnnd PF. Manual for the depression anxiety and stress scales (DASS21). Second edition. Sydney, NSW: Psychology Foundation of Australia. 1995;1-3.
Neuropsychiatric Disease and Treatment

\section{Publish your work in this journal}

Neuropsychiatric Disease and Treatment is an international, peerreviewed journal of clinical therapeutics and pharmacology focusing on concise rapid reporting of clinical or pre-clinical studies on a range of neuropsychiatric and neurological disorders. This journal is indexed on PubMed Central, the 'PsycINFO' database and CAS,

\section{Dovepress}

and is the official journal of The International Neuropsychiatric Association (INA). The manuscript management system is completely online and includes a very quick and fair peer-review system, which is all easy to use. Visit http://www.dovepress.com/testimonials.php to read real quotes from published authors. 